\title{
ACCRETION DISK WINDS DRIVEN BY THE DISK RADIATION FIELD UNDER RADIATION DRAG
}

\author{
Y. TAJIMA AND J. FUKUE \\ Astronomical Institute, Osaka Kyoiku University \\ Asahigaoka, Kashiwara, Osaka 582, Japan
}

The radiative winds from a geometrically thin accretion disk are studied. The effect of radiation drag which causes in the intense radiation fields around the accretion disk is examined recently. Then, we numerically consider the radiatively-accelerated accretion-disk winds which consist of ionized gas particles, taking into account radiation drag of the order of $v / c$.

\section{Disk Radiation Fields}

For the present purpose, we quantitatively calculate the full components of radiation fields produced by the standard accretion disk around a black hole, considering the Doppler enhancement to the order of $v / c$ (Tajima and Fukue 1997). We emphasize that there appears the azimuthal component of radiative flux, since the disk radiation field has an angular momentum.
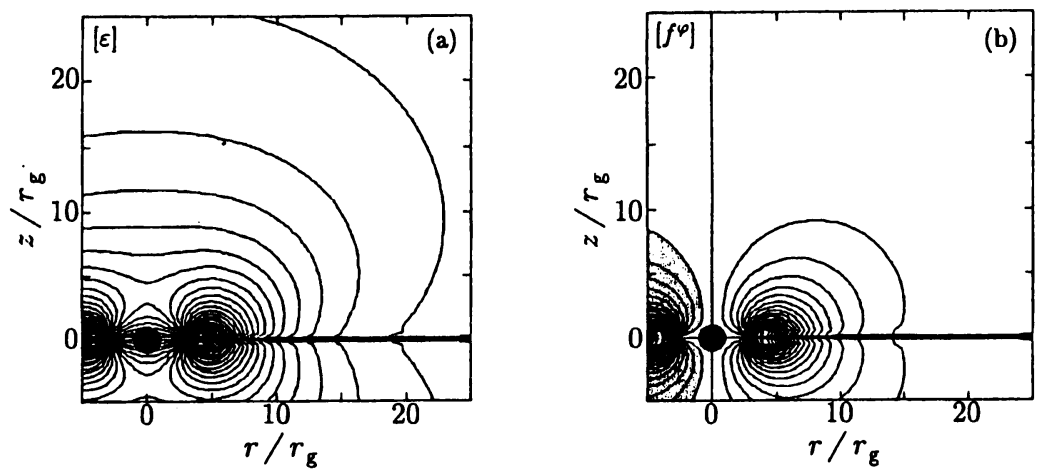

Figure 1. Examples of the contour maps of radiation fields around the accretion disk. (a) The normalized radiation energy density $\varepsilon$. (b) The azimuthal component of normalized radiative flux $f^{\varphi}$. 


\section{Motions and Escape Conditions of Particle Winds}

Using the components of disk radiation fields we examine the radiative disk winds. The acceleration in the vertical direction is suppressed, compared with the non-dragged case, due to the effect of radiation drag. When the accretion disk is less luminous, the wind cannot escape to infinity and becomes an accretion disk corona at some typical height, where the vertical gravitational force balances by the radiation force. We obtain the escape condition of the radiative disk winds. Due to radiation drag, the dragged winds become harder to blow than the winds without the drag force. Furthermore the winds tend to blow from an inner region of disks. In order for winds to blow, the disk luminosity should be larger than at least 80 percent of the Eddington luminosity, while it is 60 percent in the non-dragged case.
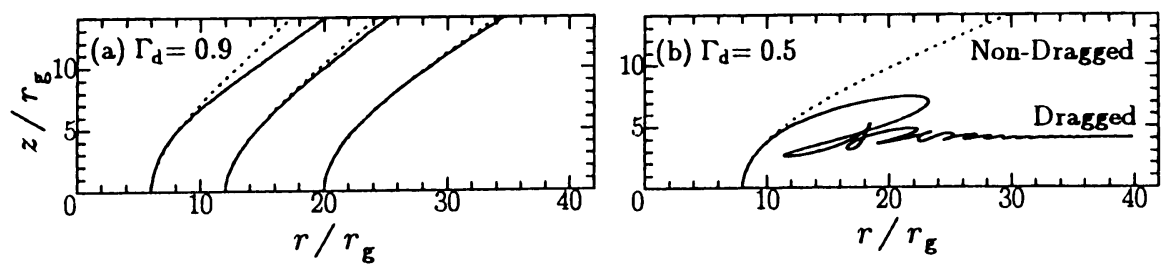

Figure 2. Examples of trajectories of the winds. The parameter $\Gamma_{\mathrm{d}}$ is the disk luminosity normalized by Eddington luminosity.

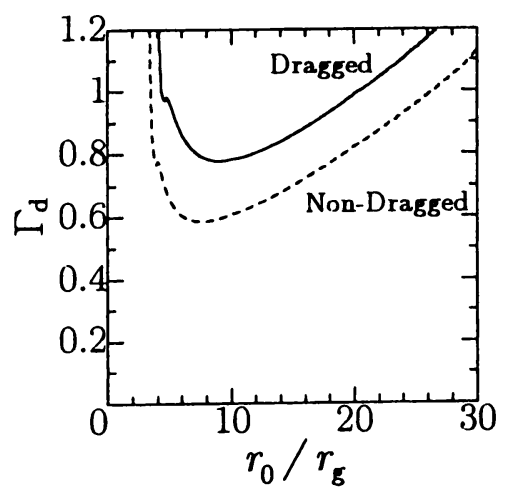

Figure 3. The escape conditions of the winds. The abscissa is the initial radius $r_{0}$ of the particle winds in units of the Schwarzschild radius $r_{\mathrm{g}}$, while the ordinate is the normalized disk luminosity $\Gamma_{d}$. When the winds have parameters above the curves in this figure, the winds can escape to the infinity and vice versa.

\section{References}

Tajima Y., Fukue J. (1997) Radiative Disk Winds under Radiation Drag II : Full Treatment, $P A S J$, submitted 